\title{
Tumor-induced VEGF-C overexpression in retroperitoneal lymph nodes in VX2 carcinoma- bearing rabbits
}

This article was published in the following Dove Press journal:

Drug Design, Development and Therapy

5 November 2015

Number of times this article has been viewed

\author{
Yong-Wen Huangl,* \\ Yun Zhoul,** \\ Chun-Yan Lan' \\ Yin Wang' \\ Yan-Ling Feng' \\ Rong-Zhen Luo ${ }^{2}$ \\ Ji-Hong Liu' \\ 'Department of Gynecology, \\ ${ }^{2}$ Department of Pathology, Sun \\ Yat-sen University Cancer Center, \\ State Key Laboratory of Oncology in \\ South China, Collaborative Innovation \\ Center for Cancer Medicine, \\ Guangzhou, People's Republic of \\ China \\ *These authors contributed equally \\ to this work
}

Objective: To establish the retroperitoneal lymph node (RLN) metastasis model of cervical carcinoma in rabbits and evaluate the relationship of vascular endothelial growth factor-C (VEGF-C) expression and the lymph node status.

Methods: Forty-eight rabbits were injected with VX2 cells or RPMI solution at muscular mucosae of the myometrium $0.5 \mathrm{~cm}$ away from the cervix. Animals were treated with or without cis-diamminedichloroplatinum(II) (cisplatin: DDP) and sacrificed on days 15, 21, and 27 post-VX2 or RPMI injections. Tumor mass and RLNs were examined histopathologically. Quantitative real-time PCR was used to examine the changes in VEGF-C mRNA expression. Levels of VEGF-C protein expression in tissues were determined using immunohistochemistry staining.

Results: Development of VX2 cervical carcinoma and the RLNs metastasis was confirmed with pathological examination. Significantly increased tumor volume was observed on days 15, 21, and 27 postinjection $(P<0.05)$. The enlargement of RLNs was found on day 21 . Expression of VEGF-C was significantly upregulated in peripheral white blood cells, tumor mass, and RLNs in an association with cancer progression. DDP resulted in a suppression of VEGF-C expression, whereas the influences on tumor mass and lymphatic metastasis were insignificant.

Conclusion: Elevated VEGF-C expressions in peripheral white blood cells and RLNs are associated with tumor progression and lymphatic metastasis. DDP treatment inhibits VEGF-C expression and fails to protect against metastatic cervical cancer.

Keywords: VEGF-C, retroperitoneal lymph nodes, VX2, cisplatin, lymphatic metastasis, peripheral blood

\section{Introduction}

Cervical cancer is the most common gynecologic malignancy with high mortality worldwide. ${ }^{1}$ Despite implantation of human papillomavirus vaccination and cytologic screening, it remains a critical issue in public health with more than 500,000 new cases and 250,000 deaths every year. ${ }^{2-4}$ Standard treatments for cervical cancer include surgical approaches in early stages and chemoradiotherapy for locally advanced ones. However, most of cervical cancers are diagnosed in advanced and/or metastatic stage, which desperately need effective treatment. ${ }^{5}$ In patients with advanced cancer, cervical cancer metastasizes to local and distal lymph nodes such as retroperitoneal lymph node (RLN). Lymph node status has been correlated with the prognosis of patient with cervical cancer. ${ }^{6,7}$ Several approaches have been evolved to evaluate the lymph node metastasis in cervical cancer, including lymphadenectomy and MRI. Nevertheless, evolution of new forms of diagnosis based on biomolecular profiling is expected to contribute to the fight against cervical cancer. ${ }^{8}$
Department of Gynecology, Sun Yat-sen University Cancer Center, State Key Laboratory of Oncology in South China Collaborative Innovation Center for Cancer Medicine, 65I Dongfeng Road East, Guangzhou, Guangdong 510060 ,

People's Republic of China

Tel +862087343102

Fax +8620873430I4

Email liujih@mail.sysu.edu.cn 
Angiogenesis and lymphangiogenesis play a critical role in the growth and metastatic spread of malignant tumor. The vascular endothelial growth factor (VEGF) family of ligands and receptor is known for its angiogenic and lymphangiogenic properties, contributing to pathogenesis of neoplasms. ${ }^{9,10}$ VEGF-C has been characterized as a lymphangiogenic and angiogenic growth factor, which is implicated in the most aggressive tumors. It promotes angiogenesis and/or lymphangiogenesis through binding to its receptor VEGFR-3 (also called Flt-4) and VEGFR-2. ${ }^{11}$ Recent studies have shown that VEGF-C and VEGFR-3 are expressed in a variety of human malignancies. ${ }^{12-14}$ Expression of VEGF-C and VEGFR-3 has been associated with poorer survival and unfavorable prognosis. ${ }^{15}$ It has also been reported that expression of VEGFR-3 is significantly correlated with the different stages of cervical carcinogenesis. ${ }^{16}$ However, the role of VEGF-C/VEGFR-3 axis in cervical cancer lymph node metastasis remains sketchy.

Animal models developed for studying cervical cancer include carcinogenic, syngeneic, and xenograft models. As rodents are relatively small, rabbit represents an ideal alternative for cervical cancer model. In the present study, we developed an in vivo model of RLN metastasis of cervical cancer by injecting rabbit tumor cells VX2 into the myometrium of New Zealand White rabbits. Using the model, the effect of cis-diamminedichloroplatinum(II) (cisplatin: DDP) on RLN metastasis was investigated. VEGF-C mRNA expression in peripheral white blood cells (WBCs) and different tissues was determined using fluorescence real-time quantitative PCR (RT-PCR). Levels of VEGF-C protein expression in tissues were determined using immunohistochemistry staining. These data may provide a theoretical basis for further studies to evaluate VEGF-C as a diagnostic factor of RLN metastasis in cervical cancer.

\section{Methods}

\section{Animals}

A total of 49 female New Zealand White rabbits (age 14 weeks, weight 1.9-2.4 kg; Huadong Xinhua Experimental Animal Center, Guangzhou, People's Republic of China) were used in this study. The animals were individually maintained in a standard cage condition and exposed daily to 12-hour light and dark cycles with free access to standard laboratory food and water. One rabbit was employed for VX2 cell preparation and the remaining animals were used to establish the in vivo model. The protocol of animal study was reviewed and approved by the Center's Animal
Welfare Committee of Sun Yat-sen University Cancer Center, Guangzhou, People's Republic of China.

\section{VX2 cells preparation}

VX2 cell line derived from rabbit squamous carcinoma was obtained from the Cell Bank of the Sun Yat-sen University. The VX2 cell preparation was performed as previously described. ${ }^{17}$ In brief, VX2 cells were suspended in $5 \mathrm{~mL}$ RPMI 1640 resulting in a cell solution of $1 \times 10^{10}$ cells $/ \mathrm{mL}$. In brief, $0.2 \mathrm{~mL}$ of the cell suspension was injected into the quadriceps femoris of rabbit. After 21 days, a tumor mass was surgically removed, washed with normal saline, and cut into pieces of $0.5-1 \mathrm{~mm}$ in diameter. The resulting mixture was vortexed and aliquoted, and the solution $\left(1 \times 10^{10}\right.$ cells $\left./ \mathrm{mL}\right)$ was transferred to a syringe using a lumbar puncture needle.

\section{Establishment of an animal model of cervical cancer with RLN metastasis}

A total of 48 animals were used and randomly divided into groups of six, namely RPMI, T15, T21, and T27. Animals were anesthetized with $3 \%$ pentobarbital sodium at $1 \mathrm{~mL} / \mathrm{kg}$ via the ear vein and then placed in a supine position. After sterilization, a mid-line incision was made on the lower abdomen. After the uterus was exposed, $0.5 \mathrm{~mL}$ of the VX2 cell solution $\left(1 \times 10^{10}\right.$ cells $\left./ \mathrm{mL}\right)$ was injected into the muscularis mucosae of the myometrium $0.5 \mathrm{~cm}$ away from the cervix. The injection site was sutured, and the wound was closed with a 1-0 suture. RPMI group received a sham surgery with an injection of $0.5 \mathrm{~mL}$ RPMI into the muscularis mucosae of the myometrium $0.5 \mathrm{~cm}$ to the cervix. For DDP treatment, animals injected with VX2 cells or RPMI solution were administrated with $4.83 \mathrm{mg} / \mathrm{kg}$ of DDP at designated time points and scarified in 4 days post-administration. The dosage was calculated using the following formula: $4.83(\mathrm{mg} /$ $\mathrm{kg})=2.1 \mathrm{mg} / \mathrm{kg}($ dose in human $) \times 2.3$ (constant for rabbit).

\section{Sample collection and pathological examination}

At each designated time point, blood samples were collected, then rabbits were sacrificed by aeroembolism. Cervix and RLN tissues were harvested, examined macroscopically and measured for the volume using the following equation: $\mathrm{V}=\mathrm{a} \times \mathrm{b}^{2} / 2$ where $\mathrm{V}=$ tumor volume, $\mathrm{a}=$ largest diameter, and $b=$ smallest diameter. Half of the tissue was placed in Trizol (Thermo Fisher Scientific, Waltham, MA, USA) and stored in liquid nitrogen and then at $-80^{\circ} \mathrm{C}$, and the other half was fixed in $10 \%$ formalin and embedded in paraffin. Sections were obtained and stained with hematoxylin and 
eosin (H\&E). The stained slides of tumor and metastatic RLN tissues were examined independently by two pathologists. Blood samples were incubated with a blood cell separation solution (Histopaque-1107, Sigma-Aldrich Co., St Louis, MO, USA) followed by centrifugation at 3,500 rpm for 10 minutes. The peripheral blood mononuclear cells were collected and stored at $-80^{\circ} \mathrm{C}$ for extraction of RNA.

\section{Real-time quantitative RT-PCR}

Total RNA was extracted from $100 \mathrm{mg}$ of tissue samples and $100 \mu \mathrm{g}$ of the peripheral blood mononuclear cells using Trizol. cDNA was synthesized using the RT-PCR kit (Promega Corporation, Fitchburg, WI, USA) following the manufacturer's instructions. The conditions for reverse transcription were $70^{\circ} \mathrm{C}$ for 5 minutes and $42^{\circ} \mathrm{C}$ for 60 minutes. The diluted cDNA $(2 \mu \mathrm{L})$ was then used for real-time PCR using the Platinum SYBR green q-PCR Super Mix-UDG (Thermo Fisher Scientific) along with the following primers, which were designed with Premier 5.0 and synthesized by the Shanghai Yingwei Jieji Co., Ltd (Shanghai, People's Republic of China): VEGF-C: 5' CCCCAAACCAGTAACAATCAGT 3' (forward), 5' CTGGCAGGGAGCGTCTAAT 3' (reverse); and GAPDH: 5' AGAGCACCAGAGGAGGACG 3' (forward), 5' TGGGATGGAAACTGTGAAGAG 3' (reverse). The conditions for the fluorescence RT-PCR were as follows: $95^{\circ} \mathrm{C}$ for 2 minutes; 45 cycles at $95^{\circ} \mathrm{C}$ for 30 seconds, $58^{\circ} \mathrm{C}$ for 30 seconds, and $72^{\circ} \mathrm{C}$ for 30 seconds; and $95^{\circ} \mathrm{C}$ for 1 minute, $58^{\circ} \mathrm{C}$ for 30 seconds, and $95^{\circ} \mathrm{C}$ for 30 seconds. The relative expression of VEGF-C was calculated as follows: $\Delta \mathrm{Ct}$ (target gene $)=\mathrm{Ct}($ target gene $)-\mathrm{Ct}(\mathrm{GAPDH}) . \Delta \Delta \mathrm{Ct}=\Delta \mathrm{Ct}($ target gene) $-\Delta \mathrm{Ct}$ (standard) mean of target gene. The relative copies of the target gene were determined as $2-\Delta \Delta \mathrm{Ct}$.

\section{Immunohistochemistry staining}

Formalin-fixed, paraffin-embedded $5 \mu \mathrm{m}$ tissue sections were stained with standard immunohistochemical methods. Sections were deparaffinized and endogenous peroxidase activity was inhibited by $0.3 \% \mathrm{H}_{2} \mathrm{O}_{2}$ in phosphate-buffered saline buffer ( $\mathrm{pH} \mathrm{7.4)} \mathrm{for} 10$ minutes. Slides were blocked with fetal bovine serum (1:10) for 20 minutes and incubated with anti-VEGF-C (dilution 1:200) at $4^{\circ} \mathrm{C}$ overnight. Resulting sides were detected with secondary biotinilated antibodies. Diaminobenzidine was used as substrate-chromogen and all slides were then counterstained with hematoxylin. Negative controls were performed without primary antibodies.

Histopathological analysis was performed by two pathologists in a blinded manner. Regions of greatest immunostaining were chosen and staining intensity was scored and presented in percentage as follows: negative $(-;<5 \%)$, weak $(1+; 5 \%-25 \%)$, moderate $(2+; 26 \%-50 \%)$, and strong $(3+;>50 \%)$.

\section{Statistical analyses}

Data are presented as means \pm standard deviation. Continuous variables among more than two groups were compared by one-way analysis of variance. When a significant difference between groups was apparent, multiple comparisons of means were performed using the Bonferroni correction with type I error. Statistical analyses were performed using SAS software version 9.2 (SAS Institute Inc., Cary, NC, USA). A two-tailed $P<0.05$ indicated statistical significance.

\section{Results}

\section{Establishment of cervical cancer with RLN metastasis in rabbits}

To investigate the role of VEGF-C in lymph node metastasis, a rabbit animal model was established by injecting VX2 cells to cervix. As shown in Figure 1, a significant increase in tumor volume was observed on days 21 and 27 postinjection $(P<0.05)$. Development of cervical cancer was confirmed by histological analysis (Figure 2). DDP is frequently used as the first-line protocols in metastatic cervical carcinoma. We examined the effect of DDP in lymph node metastasis using the VX2 carcinoma-bearing model. Administration of DDP resulted in an insignificant cervical tumor regression in the animals bearing tumor. The presence

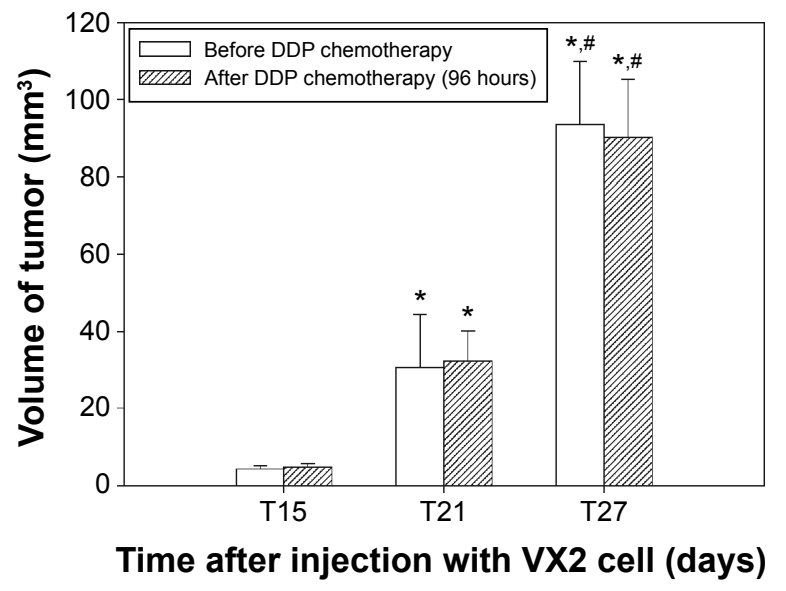

Figure I Tumor volume over time after injection with VX2 tumor cells. Notes: The size of tumors in the experimental group was determined at the indicated time points (TI5, 15 days after VX2 injection, T2I, 21 days after VX2 injection, and T27, 27 days after VX2 injection). Results represent the means \pm SD at each time point ( $n=6$ per time point). Pair-wise multiple comparisons between groups were determined using Bonferroni's correction. Indicates a statistically significant difference between the indicated group and the *TI5 group and ${ }^{*} \mathrm{~T} 2 \mathrm{I}$ group, $P<0.05$.

Abbreviations: DDP, diamminedichloroplatinum(II); SD, standard deviation. 
A
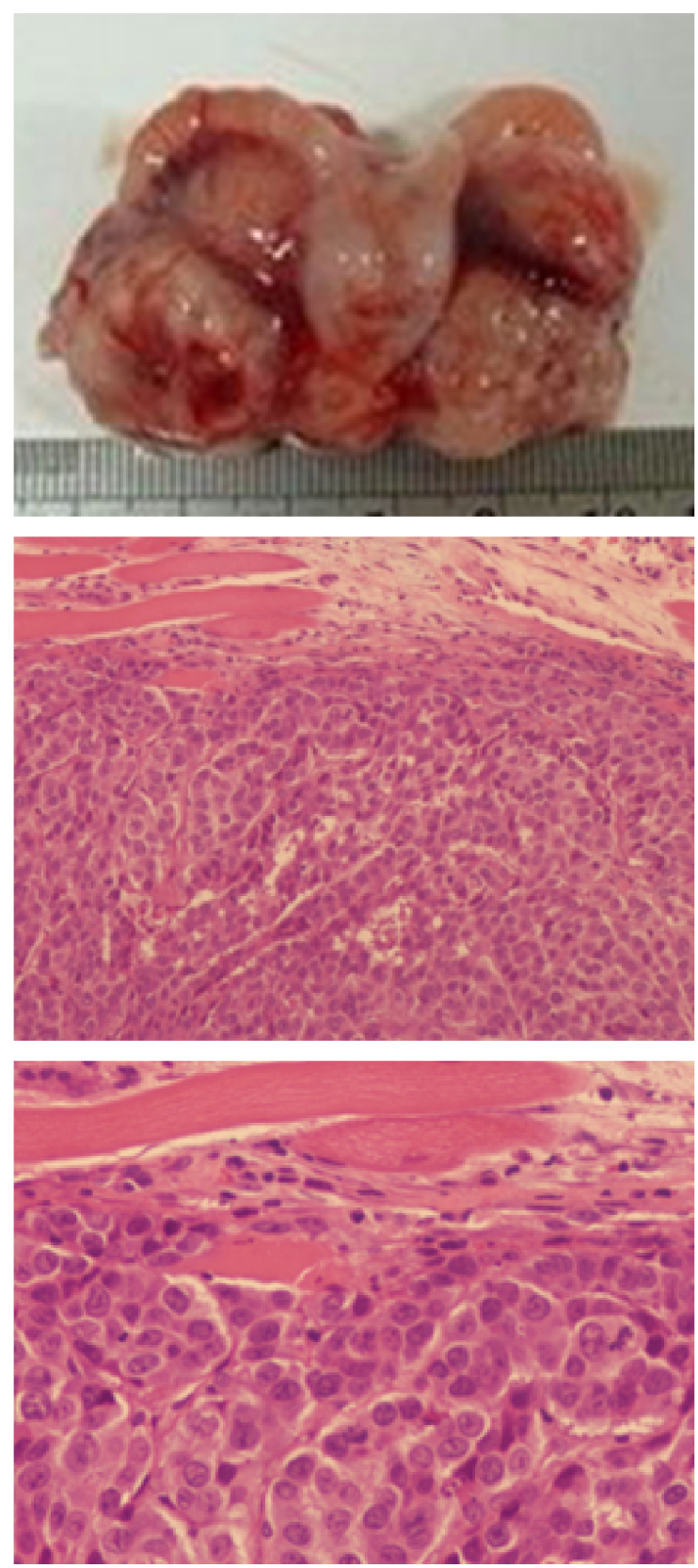

B
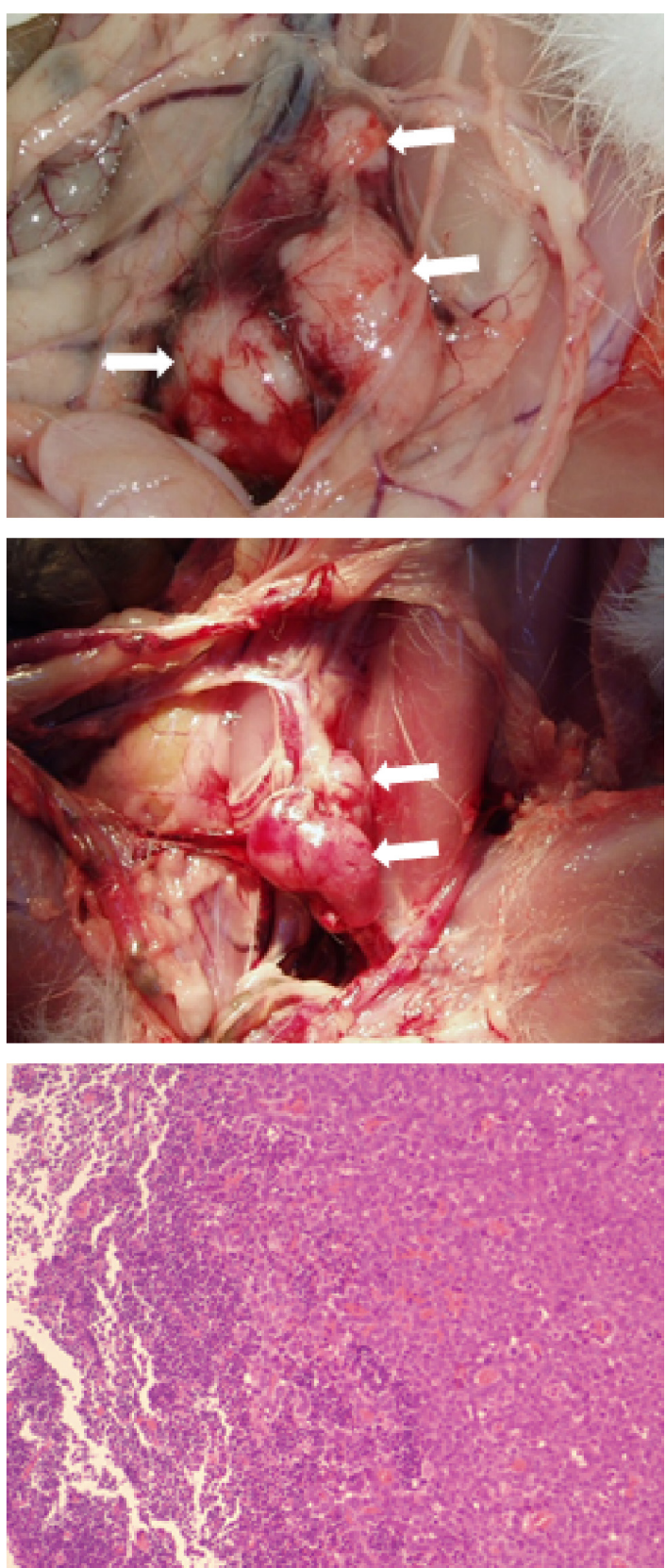

Figure 2 Representative macroscopic and histological images.

Notes: Cervix of rabbit injected with VX2 cells [(A); top to bottom: macroscopic, H\&E 100X and 400X]. RLN of rabbit injected with VX2 cells [(B); top to bottom: macroscopic, macroscopic, H\&E 40×]. Metastatic lymph nodes are indicated by arrows. Animals were sacrificed at day $2 \mathrm{I}$ and cervixes were collected and sectioned. Abbreviations: H\&E, hematoxylin and eosin; RLN, retroperitoneal lymph node.

of RLN metastasis was also assessed. Enlarged RLNs were observed macroscopically on day 21 postinjection (Figure 2). Lymphatic metastasis in rabbits bearing VX2 tumor was confirmed by histological examination.

\section{Expression profile of VEGF-C in VX2 cervical carcinoma-bearing rabbit}

We next investigated the expression levels of VEGF-C in peripheral WBCs and different metastatic tissues in VX2-bearing model. VEGF-C mRNA expression was analyzed using RT-PCR. Expression of VEGF-C in peripheral WBCs was markedly raised over time, whereas the changes in VEGF-C expression on day 15 postinjection were insignificant compared with the control (Figure 3). Treatment with DDP resulted in a significantly decreased VEGF-C expression in peripheral WBCs on day 21 postinjection (Figure $3, P<0.05$ ). Compared with the control, VEGF-C mRNA expression in the tumor tissue was markedly increased at 21 days postinjection $(P<0.05$, Figure 4A). The immunohistochemical data showed that the positive rate of VEGF-C in tumor tissues increased in 


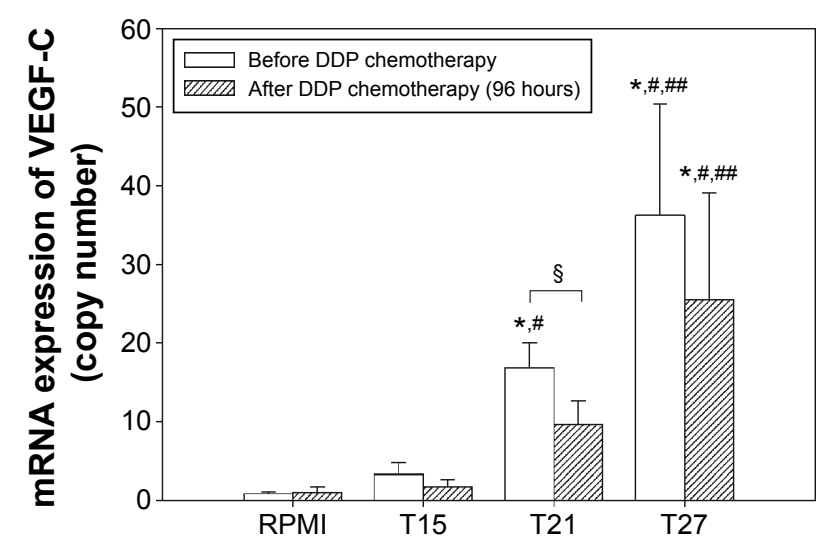

Time after injection with VX2 cell (days)

Figure 3 Differences in VEGF-C mRNA expression in peripheral white blood cells.

Notes: VEGF-C mRNA expression in the peripheral white blood cells was determined using RT-PCR over time (TI5, 15 days after VX2 injection, T2I, 2 I days after VX2 injection, and T27, 27 days after VX2 injection). Pair-wise multiple comparisons between groups were determined using Bonferroni's correction. Results represent the means $\pm S D$ at each time point ( $n=6$ per time point). Indicates a statistically significant difference between the indicated group and the *RPMl group (control), \#VX2 cell group at 15 days, and ${ }^{\prime V} \mathrm{VX} 2$ cell group at 21 days. ${ }^{8}$ Indicates a statistically significant difference between before and after DDP chemotherapy. Abbreviations: DDP, diamminedichloroplatinum(II); RT-PCR, real-time quantitative polymerase chain reaction; VEGF-C, vascular endothelial growth factor-C; SD, standard deviation.

a time-dependent manner (Figure 4B). Treatment with DDP resulted in a significantly decreased immunohistochemical positive rate compared with those without on day 27 postinjection (Figure 4B, $P<0.05$ ).

Compared to nonmetastatic RLN tissue, significantly high VEGF-C mRNA expression was observed in RLNs with metastasis (both $P<0.001$, Figure 5A). Regarding immunohistochemical positive rate of VEGF-C of RLN tissue, similar to findings in VEGF-C mRNA expression, the significantly higher positive rate was observed in RLNs with metastasis compared to those with nonmetastatic RLNs (both $P<0.001$, Figure 5B).

\section{Discussion}

Cervical cancer is the most common malignancy with poor survival rate and unfavorable prognosis in patients with lymphatic metastasis. In addition to tumor volume, lymphatic invasion and metastasis have been considered as the measures for prognosis and therapy of cervical cancer. ${ }^{18}$ It is generally accepted that lymphatic metastasis is initiated when tumor cells intravasate into lymphatic vessels and migrate to regional lymph nodes. Lymphangiogenesis is triggered by lymphangiogenic factors secreted from tumor cell and subsequently becomes the target for intravasation of tumor cells. However, the current understanding on the mechanisms underlying lymphatic metastasis is limited. In this study, we established a lymphatic metastasis model of cervical cancer in rabbit. Using this model, we demonstrated that VEGF-C expression was associated with lymphatic metastasis in cervical carcinoma. We also found that DDP suppressed VEGF-C expression in the cervical carcinoma-bearing model. However, DDP failed to interrupt lymphangiogenesis in VX-2-bearing rabbits.

The VEGF family is known to comprise five growth factors and three tyrosine kinase receptors. ${ }^{19}$ Among them, VEGF-C
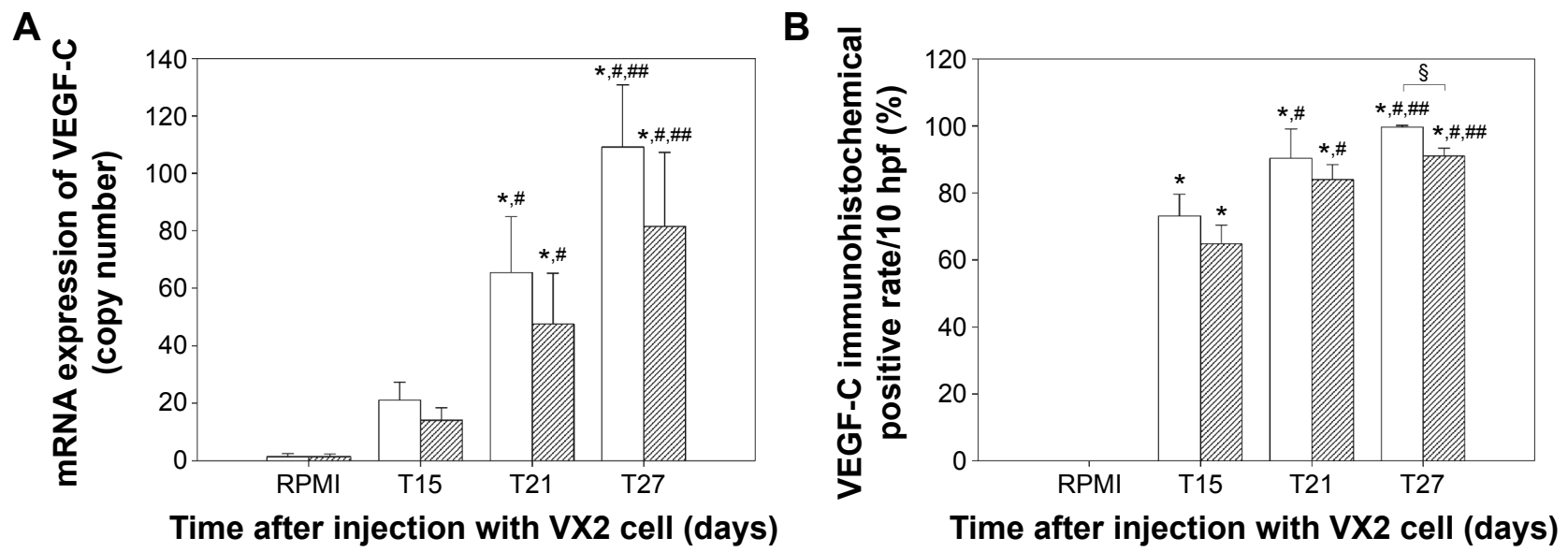

$\square$ Before DDP chemotherapy

Figure 4 Differences in VEGF-C level in the cervix and the cervical cancer.

Notes: (A) VEGF-C mRNA expression in the original tumor site was determined using RT-PCR over time (TI5, I5 days after VX2 injection, T2 I, 2 I days after VX2 injection, and T27, 27 days after VX2 injection). (B) VEGF-C immunohistochemical positive rate in the original tumor site was determined using immunohistochemical staining over time (T15, I5 days after VX2 injection, T2I, 2 I days after VX2 injection, T27, and 27 days after VX2 injection). Pair-wise multiple comparisons between groups were determined using Bonferroni's correction. Results represent the means $\pm S D$ at each time point ( $n=6$ per time point). Indicates a statistically significant difference between the indicated group and the *RPMI group (control), "VX2 cell group at 15 days, and ${ }^{\#} \mathrm{VX} 2$ cell group at 21 days. ${ }^{\circledR}$ Significance between groups of before and after DDP treatment. Abbreviations: DDP, diamminedichloroplatinum(II); RT-PCR, real-time quantitative polymerase chain reaction; VEGF-C, vascular endothelial growth factor-C; SD, standard deviation. 

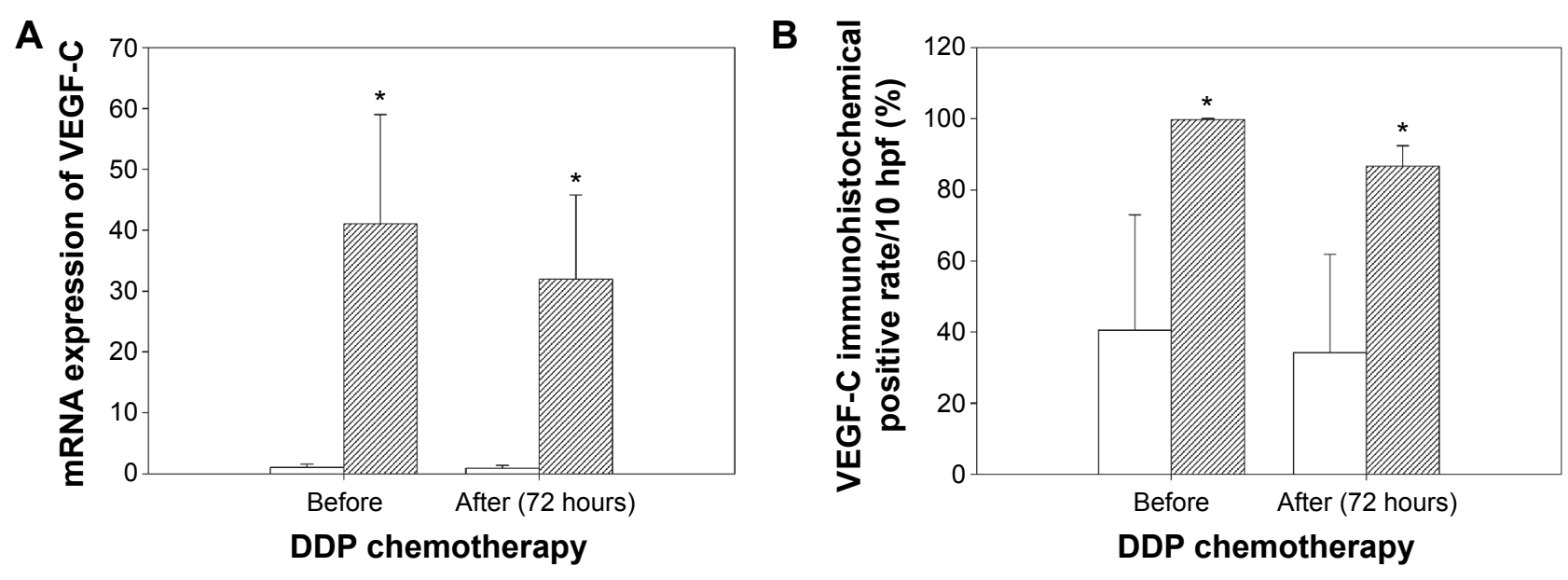

$\square$ Non-metastasized RLN WIIZ Metastasized RLN

Figure 5 Differences in VEGF-C level among the nonmetastasized RLN and metastasized RLNs, before and after DDP chemotherapy, respectively. Notes: (A) VEGF-C mRNA expression was determined using RT-PCR. (B) VEGF-C immunohistochemical positive rate was determined using immunohistochemical staining. Results represent the means \pm SD. *Indicates a statistically significant difference between nonmetastasized RLNs and metastasized RLNs.

Abbreviations: DDP, diamminedichloroplatinum; RLN, retroperitoneal lymph node; RT-PCR, real-time quantitative polymerase chain reaction; VEGF-C, vascular endothelial growth factor-C; SD, standard deviation.

is characterized as a lymphangiogenic growth factor which promotes proliferation of lymphatic endothelial cells and lymphatic vessels through an interaction with VEGFR-3 (Flt4) or VEGFR-2 (KDR). ${ }^{15}$ Excess expression of VEGF-C in tumor correlates with lymphatic metastasis and bad prognosis in several solid tumors including stomach and pancreatic cancer. ${ }^{20,21}$ Recently, it has been demonstrated that VEGF-C promotes cervical cancer metastasis through activating the RhoA/ ROCK-2 pathway. ${ }^{22}$ Our results revealed that tumor samples exhibited higher levels of VEGF-C expression than that of remote lymph node at the early stage of cervical carcinomabearing model, indicating that VEGF-C produced by cancer cells stimulate the lymphangiogenesis. A recent study has reported that mature VEGF-C increases the permeability of lymphatic vessels as well as the migration and proliferation of endothelial cells. ${ }^{23}$ Given these findings, it is implied that VEGF-C produced by primary tumor induces dilation of lymphatic vessels through interaction with activating Flt4 on the lymphatic endothelial cells. Expression of VEGF-C/ Flt-4 has been reported to correlate significantly with the different stages of cervical carcinogenesis and is considered as a specific biomarker of advanced cervical cancer clinically. Consistent with previous findings, we found that tumor growth was associated with increased levels of VEGF-C in VX2-bearing rabbits. However, further study is required to validate the relationship between tumor growth and stages of cancer in clinical aspect. VEGF-C levels in serum have been reported to be significantly elevated in cervical cancers, especially in advanced grades, compared with those in women with ovarian and endometrial cancers. ${ }^{24,25}$ In support to the findings, our data showed an elevated VEGF-C expression in peripheral WBCs, while RLN samples exhibited relatively low expression of VEGF-C. It is postulated that tumor cells initially intravasate into blood vessels through angiogenesis. In cervical cancer, VEGF-C expression has been demonstrated to associate with pelvic lymph node metastasis. However, VEGF-C mRNA expression status in RLN is not determined. Using the cervical carcinoma-bearing model, we showed that VEGF-C was overexpressed in RLNs in the late stage of the model. The results suggest that VEGF-C is involved in the lymph node enlargement in cervical cancer. Moreover, our data are in agreement with the previous study that elevation of VEGF-C expression is associated with tumor progression and lymphatic metastasis.

DDP is one of the mostly used antitumor agents against a wide variety of solid tumors. It induces toxicity against tumor cells by interaction with DNA to form DNA adducts, leading to activating several signal transduction pathways, including those involving ATR, p53, p73, and MAPK, and culminate in apoptosis activation. ${ }^{26}$ DDP is generally accepted as the standard treatment for locally advanced cervical carcinoma. For patients with metastatic cancers, efficacy of DDP is limited. In the present study, we found that DDP treatment led to a failure in tumor regression. The findings are contrary to a previous study which suggests $\mathrm{NaCl}$-added DDP suppositories as a useful local chemotherapy for endometrial carcinoma. The difference might be attributed to the different animal models used. Moreover, DDP treatment inhibits VEGF-C expression, but the changes 
in VEGF-C expression in different tissue samples following DDP treatment were statistically insignificant. It is postulated that DDP treatment induces cytotoxicity as a result of decreased mRNA expression whereas existing VEGF-C molecules remain functional in tissues. A further study with a large number of animal population is necessary to confirm the findings. The results are in line with recent studies in which survival of advanced cervical cancer was improved by co-treatment of DDP and a humanized anti-VEGF monoclonal antibody, bevacizumab. ${ }^{27}$ It is suggested that inhibition of VEGF-C production at a transcriptional level and/or interruption of VEGF-C/Flt-4 axis at a translational level has therapeutic potential for advanced cervical cancer. Further studies are required to elucidate the mechanism by which co-treatment of DDP and bevacizumab using the model established in this study.

\section{Conclusion}

A cervical carcinoma animal model with RLN metastasis was established. Using the model, we demonstrated VEGF-C overexpression in peripheral WBCs, cervical tumor mass, and metastatic RLNs. We could show that DDP reduces VEGF-C expression in metastatic cervical cancer per experimental setting. Our data also indicate that VEGF-C mRNA and protein level might serve as a new prognostic marker predicting lymphatic metastasis. Nevertheless, further clinical studies are necessarily required.

\section{Acknowledgment}

This study was funded by research grants from the Natural Science Foundation of Guangdong Province (no 10451008901006144).

\section{Disclosure}

The authors report no conflicts of interest in this work.

\section{References}

1. Ting J, Rositch AF, Taylor SM, et al. Worldwide incidence of cervical lesions: a systematic review. Epidemiol Infect. 2015;143:225-241.

2. Obel J, Souares Y, Hoy D, et al. A systematic review of cervical cancer incidence and mortality in the Pacific Region. Asian Pac J Cancer Prev. 2014; 15:9433-9437.

3. Vasilevska M, Ross SA, Gesink D, Fisman DN. Relative risk of cervical cancer in indigenous women in Australia, Canada, New Zealand, and the United States: a systematic review and meta-analysis. J Public Health Policy. 2012;33:148-164.

4. Seoud M. Burden of human papillomavirus-related cervical disease in the extended middle East and north Africa - a comprehensive literature review. J Low Genit Tract Dis. 2012;16:106-120.

5. Mendes D, Bains I, Vanni T, Jit M. Systematic review of model-based cervical screening evaluations. BMC Cancer. 2015;15:334.

6. Holman LL, Levenback CF, Frumovitz M. Sentinel lymph node evaluation in women with cervical cancer. J Minim Invasive Gynecol. 2014;21: $540-545$.
7. Gadducci A, Guerrieri ME, Greco C. Tissue biomarkers as prognostic variables of cervical cancer. Crit Rev Oncol Hematol. 2013;86: 104-129.

8. Winer I, Alvarado-Cabrero I, Hassan $\mathrm{O}$, et al. The prognostic significance of histologic type in early stage cervical cancer - a multiinstitutional study. Gynecol Oncol. 2015;137(3):474-478.

9. Sia D, Alsinet C, Newell P, Villanueva A. VEGF signaling in cancer treatment. Curr Pharm Des. 2014;20:2834-2842.

10. Kieran MW, Kalluri R, Cho YJ. The VEGF pathway in cancer and disease: responses, resistance, and the path forward. Cold Spring Harb Perspect Med. 2012;2:a006593.

11. Roskoski R Jr. Vascular endothelial growth factor (VEGF) signaling in tumor progression. Crit Rev Oncol Hematol. 2007;62:179-213.

12. Yang ZS, Xu YF, Huang FF, Ding GF. Associations of nm23H1, VEGF-C, and VEGF-3 receptor in human prostate cancer. Molecules. 2014;19:6851-6862

13. Martins SF, Garcia EA, Luz MA, Pardal F, Rodrigues M, Filho AL. Clinicopathological correlation and prognostic significance of VEGF-A, VEGF-C, VEGFR-2 and VEGFR-3 expression in colorectal cancer. Cancer Genom Proteom. 2013;10:55-67.

14. Chen JC, Chang YW, Hong CC, Yu YH, Su JL. The role of the VEGF-C/VEGFRs axis in tumor progression and therapy. Int J Mol Sci. 2012;14:88-107.

15. Su JL, Chen PS, Chien MH, et al. Further evidence for expression and function of the VEGF-C/VEGFR-3 axis in cancer cells. Cancer Cell. 2008; $13: 557-560$.

16. Van Trappen PO, Steele D, Lowe DG, et al. (2003) Expression of vascular endothelial growth factor (VEGF)-C and VEGF-D, and their receptor VEGFR-3, during different stages of cervical carcinogenesis. J Pathol. 2003;201:544-554.

17. Huang YW, Xu LQ, Luo RZ, Huang X, Hou T, Zhang YN. VEGF-c expression in an in vivo model of orthotopic endometrial cancer and retroperitoneal lymph node metastasis. Reprod Biol Endocrinol. 2013;11:49.

18. Memarzadeh S, Natarajan S, Dandade DP, et al. Lymphovascular and perineural invasion in the parametria: a prognostic factor for early-stage cervical cancer. Obstet Gynecol. 2003;102:612-619.

19. McColl BK, Stacker SA, Achen MG. Molecular regulation of the VEGF family - inducers of angiogenesis and lymphangiogenesis. APMIS 2004; 112:463-480.

20. Yonemura Y, Endo Y, Tabata K, et al. Role of VEGF-C and VEGF-D in lymphangiogenesis in gastric cancer. Int J Clin Oncol. 2005;10: 318-327.

21. Tao J, Li T, Li K, et al. Effect of HIF-1alpha on VEGF-C induced lymphangiogenesis and lymph nodes metastases of pancreatic cancer. J Huazhong Univ Sci Technol Med Sci. 2006;26:562-564.

22. He M, Cheng Y, Li W, et al. Vascular endothelial growth factor C promotes cervical cancer metastasis via up-regulation and activation of RhoA/ROCK-2/moesin cascade. BMC Cancer. 2010;10:170.

23. Persaud K, Tille JC, Liu M, et al. Involvement of the VEGF receptor 3 in tubular morphogenesis demonstrated with a human anti-human VEGFR-3 monoclonal antibody that antagonizes receptor activation by VEGF-C. J Cell Sci. 2004;117:2745-2756.

24. Landt S, Wehling M, Heidecke H, et al. Prognostic significance of angiogenic factors in uterine cervical cancer. Anticancer Res. 2011;31: 2589-2595.

25. Mathur SP, Mathur RS, Gray EA, et al. Serum vascular endothelial growth factor C (VEGF-C) as a specific biomarker for advanced cervical cancer: relationship to insulin-like growth factor II (IGF-II), IGF binding protein 3 (IGF-BP3) and VEGF-A [corrected]. Gynecol Oncol. 2005;98:467-483.

26. Boulikas T, Vougiouka M. Cisplatin and platinum drugs at the molecular level (Review). Oncol Rep. 2003;10:1663-1682.

27. Tewari KS, Sill MW, Long HJ 3rd, et al. Improved survival with bevacizumab in advanced cervical cancer. N Engl J Med. 2014;370:734-743. 


\section{Publish your work in this journal}

Drug Design, Development and Therapy is an international, peerreviewed open-access journal that spans the spectrum of drug design and development through to clinical applications. Clinical outcomes, patient safety, and programs for the development and effective, safe, and sustained use of medicines are a feature of the journal, which

has also been accepted for indexing on PubMed Central. The manuscript management system is completely online and includes a very quick and fair peer-review system, which is all easy to use. Visit http://www.dovepress.com/testimonials.php to read real quotes from published authors.

Submit your manuscript here: http://www.dovepress.com/drug-design-development-and-therapy-journal 